\title{
The Fused Deposition Modeling 3D Printing
}

\author{
Longwei Yan, Huichao Sun*, Xingtian Qu and Wei Zhou \\ School of Mechanical Science and Engineering, Jilin University, China \\ ${ }^{*}$ Corresponding author
}

\begin{abstract}
Introduction-Fused Deposition Modeling (FDM) is one of the most widely used additive manufacturing processes for fabricating prototypes and functional parts in common engineering plastics. From the view of the control structure, 3D printer based on the FDM technology is divided into two layers: the host computer and the bottom control. The main working parts of the FDM 3D printer are nozzle mechanism, wire feeder, motion mechanism, heating work mechanism and working platform.
\end{abstract}

Keywords-3D printing; fused deposition modeling; host computer; bottom control

\section{3D PRINTING-NEW EPOCH OF CREATION}

3D printers are the first wave of a new generation of intelligent machines. They are capable of designing, manufacturing, repairing and recovering of other machines, and even adjusting and improving other machines, including their own [1].

The goal of the fourth industrial revolution is to achieve plant intelligence. In the process of actual production, dispersed intelligent manufacturing devices are closely bound together through the form of network, thus these devices possess more open, more positive communication system structure, more dynamic and flexible, and as a result, they can discover more optimization possibilities. For products, highly flexible production plants can meet customer requirements for product individuation, diversification and constantly changing [2]. For factories, employees can possess more flexible working hours. During this period, the Rapid Prototyping Manufacturing, otherwise known as 3D printing, based on CAD/CAM has rapidly became a social and technological hotspot.

\section{THE DEFINITION OF 3D PRINTING}

Through the use of layer by layer accumulating of specific material to produce three-dimensional physical model, 3D printing is an innovative manufacturing technology which takes advantage of the CAD model. There is no need to develop new mould in the entire product manufacturing process, and the mould obtained by three-dimensional solid modeling of computer can print products directly, so it is possible to achieve product rapid manufacturing.

3D printing is a technology which mainly uses powder metal or plastic and other materials to bond, and it constructs objects through layer by layer printing. 3D printing often used to be applied to manufacturing model in mould manufacturing, industrial designing and other fields, and it is now gradually used for direct manufacturing of products [3].

\section{THE PROCESS OF 3D PRINTING}

Before employing 3D printing to manufacture model, there is a need to design three-dimensional model of the printout on computer. At present, the computer software can slice the three-dimensional model and also is capable of designing the layer by layer printing file. The 3D printing workflow is shown in Fig. 1. 3D printer controls its nozzle position under the instruction of the design document. The nozzle spurts solid metal powder or molten thermoplastic material which will solidify into a plane sheet of metal or thermoplastic on the working platform. After the first thin layer solidified, the 3D printer nozzle returns to the original point of printing according to the design document, and another thin layer is formed on the first layers outside. Repeat the above process, and finally complete the three-dimensional printing through overlying layer by layer[4].

\section{Fused Deposition 3D printing}

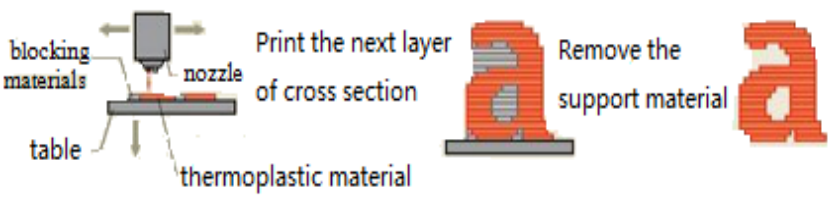

FIGURE I. THE WORKFLOW OF 3D PRINTING

\section{The Structure of THE Fused Deposition Modeling 3D PRINTER}

The FDM 3D printer which is a rapid prototyping printer based mainly on printing plastic with plasticity is the most rapid development and the most mature technology in the current 3D printing market, and it is also the most widely applied 3D printer currently. From the view of the control structure, 3D printer based on the FDM technology is divided into two layers: the host computer and the bottom control. The host computer mainly runs three-dimensional designing software, slicing software, printing control software and so on. The bottom control includes embedded microcontroller, main board, stepping motor, motor driver, limit switch, thermoplastic material extruder, printing platform, temperature sensor and so on[5]. The main working parts of the FDM 3D printer are nozzle mechanism, wire feeder, motion mechanism, heating work mechanism and working platform.

\section{A. The intelligent nozzle of the Fused Deposition Modeling 3D printer}

In the process of the FDM 3D printing, after being heated and melted in the heating chamber of the nozzle, the filamentous thermoplastic material enters the nozzle under the action of the piston rod of the non-melted material, and then under a certain pressure, the nozzle squeezes out the 
thermoplastic filament with a predetermined diameter so as to form a finished product with high precision. The intelligent nozzle of the FDM 3D printer is shown in Fig. 2. The intelligent nozzle is mainly composed of feeding pipe, heating pipe, fin, thermistor, nozzle and exhaust fan.

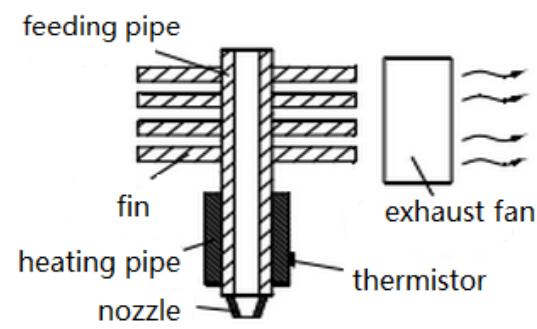

FIGURE II. THE INTELLIGENT NOZZLE OF THE FUSED DEPOSITION MODELING 3D PRINTER

In the process of working, the printing raw material of the FDM 3D printer gets into the feeding pipe through the wire feeder, and then enters the heating pipe through the transition pipe, and then the thermoplastic material is heated in the heating pipe which regulates heating temperature with feedback regulation via thermistor. Squeezed by the nonmelting material, the material of heating and melting parts squeezes out the thermoplastic filament of predetermined diameter from the nozzle. The influence of the nozzle structure on the FDM printing is mainly the internal flow structure of the nozzle and the control of heating temperature of the nozzle and also the different pressure difference in each flow generated by flow fields in different flows. The temperature of the nozzle affects various performance of material, such as cohesiveness, accumulation resistance, wire flow, extruded wire width and so on. For one thing, if the temperature is too slow, it will lead to partial solid material, as a result, too large viscosity of material will affect the velocity of wire and even the block nozzle; For another thing, if the temperature is too high, it will lead to partial liquid material, as a result, the wire material appears sallow and increasing mobility and also too fast extruded speed, which can't control the wire precision accurately. The nozzle flow mainly applies Teflon material which has acid-base resistance and various organic solvent resistance characteristics and even almost insoluble in all solvents. [6]

\section{B. The Wire Feeding Mechanism of the FDM 3D Printer}

In the process of the FDM 3D printing, the wire feeding mechanism delivers the printing raw material for printer's nozzle. In order to ensure the accuracy of the forming and the stability of the printed object, the wire feeding mechanism is required stable and reliable. The raw materials of the FDM printing are generally Nylon glass fiber, PLA plastic, ABS plastic. The diameter of the raw material is generally $1 \sim 2 \mathrm{~mm}$, but the nozzle's diameter is only about $0.2 \sim 0.5 \mathrm{~mm}$, thus the difference between the raw material diameter and the nozzle' $s$ diameter requires that the nozzle has a certain pressure and the molten material can be extruded at a certain speed. The wire feeder mechanism formed mainly by two DC motors controls the wire feed speed and the open-close condition at any moment with the cooperation of $\mathrm{D} / \mathrm{A}$ control module. To ensure the stability and reliability of the wire feeding and avoid the broken wire or the accumulated burl, the wire feeding mechanism and the nozzle adopt a way of push-pull combination [7].

The wire feeding mechanism of the fused deposition printer is shown in Fig. 3. The stepping motor is generally a $1 / 16$ stepping motor of 1.8 degrees and its wire feeding speed is $0 \sim 300$ per second. In order to improve the silk yielding precision and the printing speed, there have requirements to improve the driving force of driving wheels to wire materials and the pressure of the non-melted wire materials to melt in the channel. At present, the methods of improving the driving force of the driving wheels to the wire materials mainly are increasing the power of stepping motor, enlarging the friction of driving wheels to wire materials and changing the contact type of driving wheels to wire materials. There is a need to find a balance between the heating speed of the heating chamber and the wire feeding speed of the wire feeding mechanism.

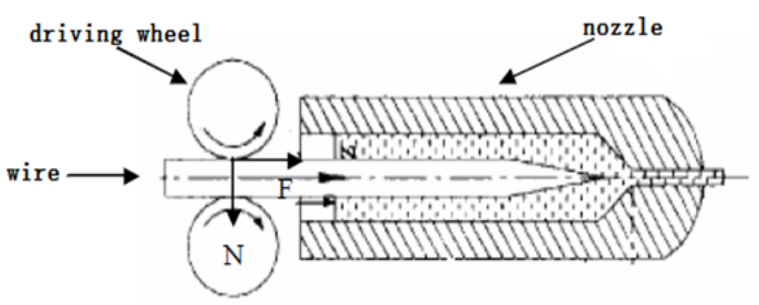

FIGURE III. THE WIRE FEEDING MECHANISM OF THE FUSED DEPOSITION MODELING 3D PRINTER

\section{The Moving Mechanism of the Fused Deposition Modeling $3 D$ Printer}

The moving mechanism of the FDM printer consists of the plane motion of $\mathrm{X}$ and $\mathrm{Y}$ axes and the vertical motion of $\mathrm{Z}$ axis. The principle of the $3 \mathrm{D}$ printing is to convert arbitrary complex three-dimensional model into plane figure's accumulation. At present, the moving mechanism of the FDM 3D printer is mainly divided into the frame-type and the delta-type. The 3D printer is mainly to transform the three-dimensional printing into two-dimensional printing, therefore no longer require the three-axes and more axes linkage of 3D printer, only need to complete the two-dimensional linkage, which greatly simplifies the printer's motion control. The FDM 3D printer of frame-type is shown in Fig. 4. The $\mathrm{X}-\mathrm{Y}$ axes linkage complete the plane printing and the $\mathrm{Z}$-axis drives the working platform to achieve the feed of the direction of height, and then complete the accumulation through layer by layer. The FDM 3D printer of delta-type is shown in Fig. 5. The three stepping motors are installed on the guide. The stepping motor drives the synchronous belt on slider through the synchronous belt wheel on the motor's light bars, therefore the rotary motion of motor shaft is transformed into rectilinear motion. The synchronous belt on slider drives the slider to move up and down on the guide. The delta-type 3D printer which transforms the Rectangular coordinates into Cartesian space coordinates improves the printing height and saves the printing space. The differences of delta-type 3D printer from other printer structures are that its stepping motors of the wire feeding mechanism are fixed on triangle frame, and the stepping motors do not move simultaneously with the nozzle, so that it 
extremely decreases the inertia of the nozzle, which has a great help to improve the printing speed and the control precision.

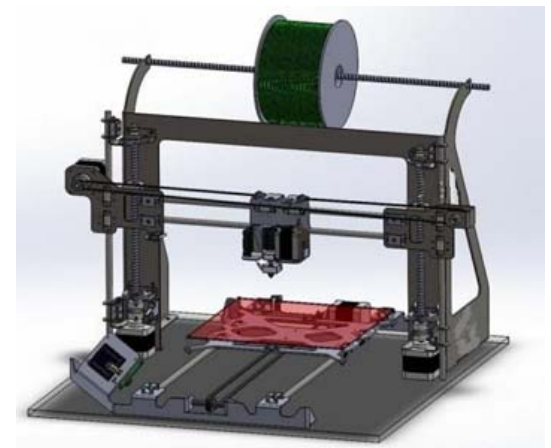

FIGURE IV. THE FUSED DEPOSITION MODELING 3D PRINTER OF FRAME-TYPE

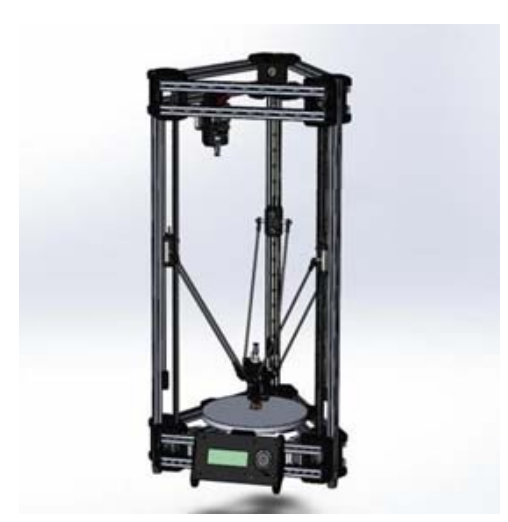

FIGURE V. THE FUSED DEPOSITION MODELING 3D PRINTER OF DELTA-TYPE

\section{The Temperature Control Mechanism of the Fused Deposition Modeling 3D Printer}

The process of the FDM 3D printing is mainly to heat and melt the raw material and then print in a plane by the nozzle, and finally the product is fixed and formed through layer by layer accumulating. Therefore, in the process of printing the heating temperature of the nozzle and the modeling temperature of the working platform are demanded to control. The temperature control mechanism is shown in Fig. 6. The heating chamber of the nozzle is heated by the heating rod and the temperature controller. The heating rod is responsible for heating, and the temperature controller controls the heating temperature and through the thermistor controls the heating circuit by timely feedback. The regulation of nozzle temperature is mainly to control the heating speed and the feedback speed. The materials of the FDM 3D printing mainly are PLA plastic and ABS plastic, and the melting point of these two materials is all about $170^{\circ}$. In order to shorten the printing time and improve the printing precision, the heating speed and the over-temperature feedback ability need to be improved. The temperature controlling of working platform is mainly to avoid the warp in the process and molding defects when the product is forming. So, it is needed to stabilize the working platform in a relatively stable temperature, which can be achieved by a long time control of thermistor at a definite temperature range.

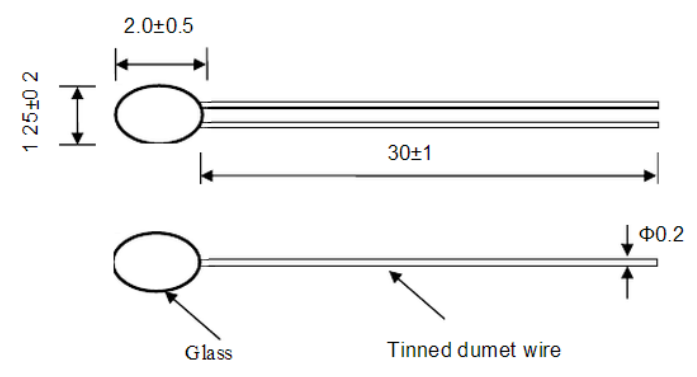

FIGURE VI. THE TEMPERATURE CONTROL MECHANISM

\section{The DeVelopment TREnd of the Fused Deposition MODELING TECHNOLOGY}

Fused Deposition Modeling is one of the most widely used additive manufacturing processes for fabricating prototypes and functional parts in common engineering plastics. It is possible that industrial printers will be used by online service providers or copy shops. Consumers could upload their designs to these service providers. The products subsequently will be printed in 'fabrics' with many 3D printers. Furthermore, 3D printing will contribute to a circular economy, where printed products will be recycled and fully reproduced again. This work was financially supported by the National Natural Science Foundation of China (51135006).

\section{REFERENCES}

[1] Lipson, Melba Kurman.2013. Fabricated: The New World of 3D Printing[M]. China: CITIC Press.

[2] Zhang Haiping.2014. The Fourth Industry Revolution Fourth Industry Revolution[J]. Fluid Power Transmission and Control. 2014(2).

[3] Zhao Mingcheng.2013. The research on digital architecture design and fabrication or construction[D]. Hunan University.

[4] Yu Meng.2007. The research on fused deposition molding materials and support materials[D]. Huazhong University of science and technology.2007.

[5] Cui Wangrui, Li Yuxin, Li Fukun, Yu Hang, Jiang Heping.2014. 3D printer architecture research[J]. Computer CD software and application.2014(16).

[6] Wu Yuqi, Gu Jun. The middle blank automatic transmission design of solar panel[J]. Technology Wind.2012(10).

[7] Wang Tiantian. The research and design of FDM wire feeding institutions[D]. Huazhong University of science and technology.2007. 